Pacific

Journal of

Mathematics

COMBINATORIAL EXCURSIONS IN MODULI SPACE

Roger W. Barnard and G. Brock Williams 


\title{
COMBINATORIAL EXCURSIONS IN MODULI SPACE
}

\author{
Roger W. BARnARd AND G. Brock Williams
}

\begin{abstract}
Given an abstract triangulation of a torus, there is a unique point in moduli space which supports a circle packing for this triangulation. We will describe combinatorial deformations analogous to the process of conformal welding. These combinatorial deformations allow us to travel in moduli space from any packable torus to a point arbitrarily close to any other torus we choose. We also provide two proofs of Toki's result that any torus can be transformed into any other by a conformal welding and compute the maps necessary to accomplish the welding.
\end{abstract}

\section{Introduction.}

A circle packing is a configuration of circles with a prescribed pattern of tangencies. The interplay between the combinatorial "pattern" and the geometry provided by the circles has generated intense interest in recent years. As a result, a discrete version of complex analysis based on maps between circle packings has now emerged. The seeds of a discrete Teichmüller theory have been sown, but it is still in the very early stages of development.

It has been understood that the underlying combinatorial structure of a circle packing determines its geometry. For packings on surfaces, the combinatorics determine a unique point in moduli space on which the packing "lives" [4]. As we will see in Section 5, changing the combinatorics generally changes the point in moduli space, but the precise mechanism remains very mysterious. Our goal then is to develop some controlled combinatorial procedure for which we not only understand the resulting movement in moduli space, but which we can actually use to drive us to a specified target.

Toki [26] proved that for the torus, any point in moduli space can be deformed into any other by a conformal welding. That is, any torus can be cut open along an appropriate geodesic and then glued (unevenly) back together to form any other torus. After introducing some background material in Section 2, we present another proof of Toki's result in Section 3. Our approach allows us to explicitly compute the welding maps involved.

Theorem 1.1. Consider a point $\tau \in \mathbb{H}$. If $\tau^{\prime} \in\{z \mid 0<\operatorname{Im} z \leq \operatorname{Im} \tau\}$, then there is a conformal welding along any vertical geodesic which will transform 
$R_{\tau}$ to $R_{\tau^{\prime}}$. Conversely, if $\tau^{\prime} \in\{z \mid \operatorname{Im} z \geq \operatorname{Im} \tau\}$, then there is a conformal welding along any horizontal geodesic which will transform $R_{\tau}$ to $R_{\tau^{\prime}}$.

In Section 6, we describe a combinatorial deformation analogous to welding. Packings are cut open along a geodesic, then reconnected in a different pattern than before. In Section 7 we prove that these discrete weldings approximate their classical counterparts.

Theorem 1.2. Fix any abstract triangulation $\mathcal{K}$ of a torus and any point $\tau^{\prime} \in \mathbb{H}$. Then combinatorially deforming $\mathcal{K}$ by hex refinement and combinatorial welding along an appropriate geodesic produces packable surfaces which converge in the Teichmüller metric to $R_{\tau^{\prime}}$.

Thus we can prescribe the combinatorial changes necessary to move in moduli space from any packable surface to a point arbitrarily close to any other surface we choose. This also gives a second proof of Toki's theorem using only circle packing techniques. Moreover, our proof holds for any bilipschitz welding map, not only the ones needed for our excursions in moduli space. Thus we have a discrete method for approximating the surface created by any bilipschitz welding.

Our procedure allows for several arbitrary choices in the small scale changes made to the complexes. However, the welding process is remarkably stable, forcing these variations in the construction to disappear in the limit.

Theorem 1.2 also yields a genus one version of Brooks's density result for compact surfaces [8]. Not only are the packable tori dense, but any one packable torus generates a dense packable family by welding. A similar result for hyperbolic surfaces was given by the second author in [28] using earthquake deformations.

Finally, we would like to thank the referee for several very helpful suggestions.

\section{Teichmüller theory.}

2.1. Riemann surfaces and their deformation spaces. We begin with a brief review of Riemann surfaces and Teichmüller spaces. Some of the many excellent references include $[\mathbf{1 0}, \mathbf{1 1}, \mathbf{1 5}, \mathbf{1 8}, \mathbf{1 3}]$.

Definition 2.1. A Riemann surface is a one complex-dimensional manifold with charts whose overlap maps are conformal. A maximal collection of such charts define a conformal structure.

Surfaces $R_{1}$ and $R_{2}$ are said to be conformally equivalent if there is a conformal homeomorphism $f: R_{1} \rightarrow R_{2}$. The equivalence classes of surfaces of the same topological type as $R_{1}$ constitutes the moduli space of $R_{1}$. For many purposes, however, we need an equivalence relation that keeps track of more information. Equivalent surfaces should not only share the same 
conformal structure but generators of their fundamental groups should also correspond.

Definition 2.2. Let $R$ be a Riemann surface and $\Sigma$ a collection of canonical generators of $\pi_{1}(R)$. $\Sigma$ is then called a marking for $R$. Two markings are equivalent if they differ only by the choice of basepoint.

Definition 2.3. Two marked Riemann surfaces $(R, \Sigma)$ and $\left(R^{\prime}, \Sigma^{\prime}\right)$ are equivalent if there is a conformal map $f: R \rightarrow R^{\prime}$ for which the marking $f_{*}(\Sigma)$ is equivalent to $\Sigma^{\prime}$. The Teichmüller space of $R_{1}$ is the set of these equivalence classes.

An alternative definition can be given using maps from a reference surface.

Definition 2.4. Quasiconformal maps $f_{1}$ and $f_{2}$ of a Riemann surface $R$ are Teichmüller equivalent if $f_{2} \circ\left(f_{1}\right)^{-1}$ is homotopic to a conformal map.

Proposition 2.5. Fix a reference Riemann surface $R$ and suppose $f_{1}$ and $f_{2}$ are quasiconformal maps from $R$ to $R_{1}$ and $R_{2}$, respectively. Then $R_{1}$ and $R_{2}$ are equivalent in Teichmüller space if and only if $f_{1}$ and $f_{2}$ are equivalent mappings.

There is a natural metric on Teichmüller space determined by how nearly conformal a homeomorphism which respects the markings may be. More specifically, the distance between surfaces $R_{1}=f_{1}(R)$ and $R_{2}=f_{2}(R)$ is given by

$$
\frac{1}{2} \log K^{*}
$$

where $K^{*}$ is the infimum of the dilatation of $g_{2} \circ\left(g_{1}\right)^{-1}$ with $g_{i}$ equivalent to $f_{i}, i=1,2$. This infimum is attained by a unique Teichüller mapping.

The collection of homotopy classes of diffeomorphisms of $R$ is called the mapping class group of $R$. It acts on the Teichmüller space of $R$ transforming one marking into another. Teichmüller space modulo the mapping class group is precisely the moduli space. Thus the Teichmüller metric induces a metric on moduli space.

2.2. Tori. Consider a point $\tau \in \mathbb{H}$ and the group $\Gamma_{\tau}$ of translations of $\mathbb{C}$ generated by $z \mapsto z+\tau$ and $z \mapsto z+1$. The quotient surface $R_{\tau}=\mathbb{C} / \Gamma_{\tau}$ is a torus. A fundamental region for $R_{\tau}$ is the parallelogram $\Omega_{\tau}$ with vertices at $0,1, \tau$, and $1+\tau$.

Such a torus has two families of geodesics corresponding to its generators $z \mapsto z+1$ and $z \mapsto z+\tau$. The horizontal geodesics are the projections of horizontal lines, that is, lines parallel to the translation $z \mapsto z+1$. Similarly, the vertical geodesics are the projections of lines parallel to the translation $z \mapsto z+\tau$.

Any of the tori $R_{\tau}$ are topologically identical, but their conformal structures change with $\tau$. In fact the Teichmüller space for the torus is isometric 
to $\mathbb{H}$ with the hyperbolic metric. The mapping class group is isomorphic to $\mathrm{PSL}_{2} \mathbb{Z}$, and the moduli space is isometric to $\mathbb{H} / \mathrm{PSL}_{2} \mathbb{Z}$.

\section{A moduli space trip in a classical vehicle.}

3.1. Conformal welding. Suppose $R$ is a Riemann surface and $\gamma$ a simple closed geodesic. If we cut $R$ open along $\gamma$, we obtain a bordered surface $\bar{R}$ with geodesic boundary $\operatorname{arcs} \gamma^{+}$and $\gamma^{-}$. Notice that each point $z \in \gamma$ splits into points $z^{+} \in \gamma^{+}$and $z^{-} \in \gamma^{-}$.

Definition 3.1. If $\bar{R}$ is a bordered Riemann surface with geodesic boundaries $\gamma^{+}$and $\gamma^{-}$as above and $\varphi: \gamma^{+} \rightarrow \gamma^{-}$, then a conformal welding for $\varphi$ is a map $f$ from $\bar{R}$ to some surface $R^{\prime}$ which is conformal off $\gamma^{+} \cup \gamma^{-}$and satisfies the welding property

$$
f \varphi\left(z^{+}\right)=f\left(z^{-}\right)
$$

where $z^{+} \in \gamma^{+}$and $z^{-} \in \gamma^{-}$are copies of the same point $z \in \gamma$.

From a topological viewpoint, gluing $\gamma^{+}$and $\gamma^{-}$together is relatively uninteresting. The new surface $R^{\prime}$ is topologically equivalent to the original surface $R$, and the welding curve $\gamma^{\prime}=f\left(\gamma^{+}\right)=f\left(\gamma^{-}\right)$is homotopic to $\gamma$. From a conformal point of view, however, $R$ and $R^{\prime}$ may be dramatically different surfaces. The welding map $\varphi$ stretches and compresses $\gamma^{+}$as it attaches it to $\gamma^{-}$. The new "seam" $\gamma^{\prime}$ must absorb this distortion, bending and warping as the competing metric forces do battle. See Figure 1.

The existence of a conformal welding allows us to define coordinate charts on $R^{\prime} \backslash \gamma^{\prime}$ using the charts on $R$. That is, if $\left\{\phi_{\alpha}\right\}$ is the conformal structure on the bordered surface $\bar{R}$, then $\left\{\phi_{\alpha} f^{-1}\right\}$ is a conformal atlas on $R^{\prime} \backslash \gamma^{\prime}$. As a result, the metric distortion between $\bar{R}$ and $R^{\prime}$ is completely captured in the geometry of $\gamma^{\prime}$.

Notice that if $g: R^{\prime} \rightarrow R^{\prime \prime}$ is conformal, then $g f$ is another conformal welding for $\varphi$. If every welding for $\varphi$ is of this form, then $\varphi$ determines a unique point in moduli space. Oikawa [20] proved that unique weldings exist for locally quasisymmetric maps (that is, maps which can locally be extended to quasiconformal maps) gluing the sides of infinite strips to form a cylinder. Since a torus is covered by a cylinder, locally quasisymmetric welding maps on a torus can be lifted to periodic locally quasisymmetric welding maps on a cylinder. Oikawa's result can then be restated as follows.

Theorem 3.2. If $\varphi$ is a locally quasisymmetric welding map on a torus, then welding by $\varphi$ determines a unique point in moduli space.

Beyond uniqueness, we are interested in the geometry of tori deformed by welding. The following result appears in a paper of Toki [26]. Toki's proof, however, filters the welding through the exponential map and does not provide a simple description of the welding $f$. Our proof is similar in 

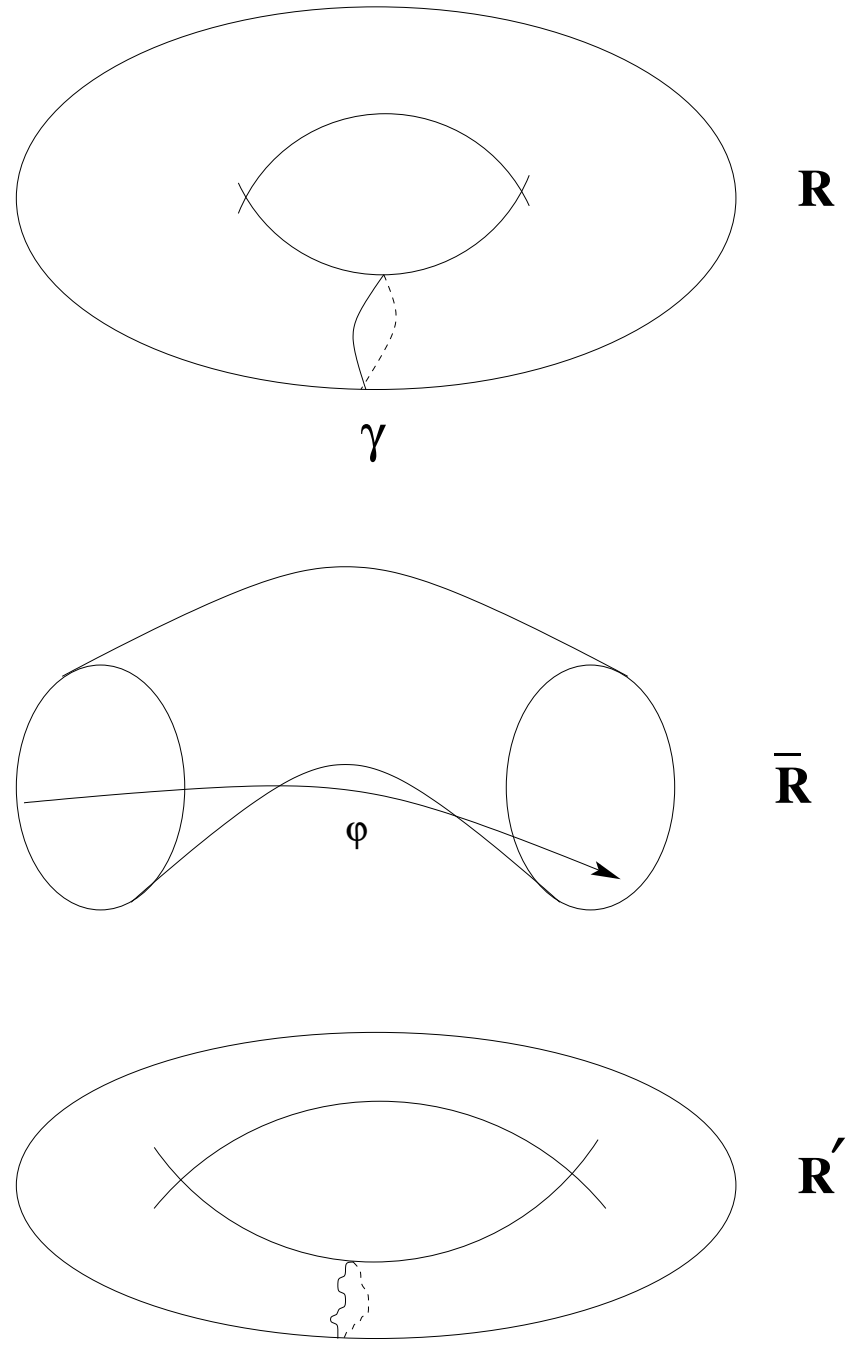

Figure 1. A torus before, during, and after welding. Notice that before welding (top) the torus is much "fatter" than after the welding (bottom). The global geometric distortion is also reflected in the distortion of the image of the geodesic $\gamma$.

spirit, but yields an explicit description of the deformation needed to convert one torus to another. In Section 7, we present a second proof using discrete maps induced by circle packings.

Theorem 1.1. Consider a point $\tau \in \mathbb{H}$. If $\tau^{\prime} \in\{z \mid 0<\operatorname{Im} z \leq \operatorname{Im} \tau\}$, then there is a conformal welding along any vertical geodesic which will transform 
$R_{\tau}$ to $R_{\tau^{\prime}}$. Conversely, if $\tau^{\prime} \in\{z \mid \operatorname{Im} z \geq \operatorname{Im} \tau\}$, then there is a conformal welding along any horizontal geodesic which will transform $R_{\tau}$ to $R_{\tau^{\prime}}$.

Proof. First suppose $0<\operatorname{Im} \tau^{\prime} \leq \operatorname{Im} \tau$ and choose a geodesic $\gamma$ of $R_{\tau}$ corresponding to the translation $z \mapsto z+\tau$. Recall that a fundamental region for $R_{\tau}$ and $R_{\tau^{\prime}}$ is given by the parallelogram $\Omega_{\tau}$ having vertices $0,1, \tau, 1+\tau$. By an appropriate choice of basepoint, we can assume $\gamma$ lifts to the left and right sides of $\Omega_{\tau}$. We will label these sides $\widetilde{\gamma}^{+}$and $\widetilde{\gamma}^{-}$, respectively.

Similarly, $R_{\tau^{\prime}}$ lifts to a fundamental region given by the parallelogram $\Omega_{\tau^{\prime}}$ with vertices $0,1, \tau^{\prime}, 1+\tau^{\prime}$. In general, $\Omega_{\tau}$ and $\Omega_{\tau^{\prime}}$ will have different conformal moduli (as quadrilaterals) and hence no conformal map between them. However, if we distort $\Omega_{\tau^{\prime}}$, we can obtain a fundamental region for $R_{\tau^{\prime}}$ whose conformal modulus equals that of $\Omega_{\tau}$.

By decreasing the angle at $\tau^{\prime}$ and 0 and simultaneously increasing the angle at $\tau^{\prime}+1$ and 1 , we bend the middle of $\Omega_{\tau^{\prime}}$ to the right. See Figure 2 .
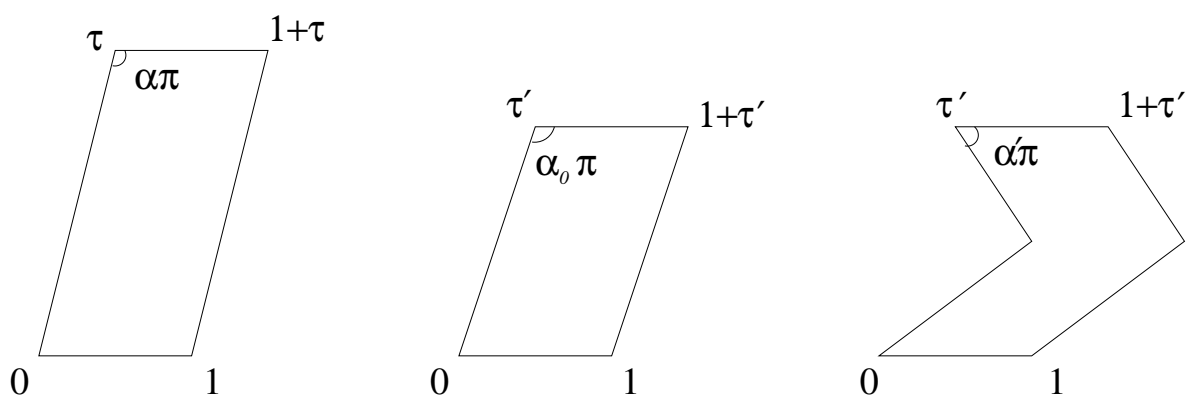

Figure 2. The quadrilateral $\Omega_{\tau}$ (left) has a smaller modulus than $\Omega_{\tau^{\prime}}$ (middle). By sliding the "middle" of $\Omega_{\tau^{\prime}}$ to the right, we can lower the modulus of $\Omega_{\tau^{\prime}}\left(\alpha^{\prime}\right)$ (right).

If $\alpha_{0} \pi=\pi-\operatorname{Arg}\left(\tau^{\prime}\right)$ is the angle in $\Omega_{\tau^{\prime}}$ at $\tau^{\prime}$, then for $0<\alpha^{\prime} \leq \alpha_{0}$, let $\Omega_{\tau^{\prime}}\left(\alpha^{\prime}\right)$ be the 6 -gon with vertices at $0, \tau^{\prime}, 1+\tau^{\prime}$, and 1 , angle $\alpha^{\prime} \pi$ at 0 and $\tau^{\prime}$, and angle $\left(1-\alpha^{\prime}\right) \pi$ at 1 and $1+\tau^{\prime}$

Notice that $\bmod \left(\Omega_{\tau^{\prime}}\left(\alpha_{0}\right)\right)=\bmod \left(\Omega_{\tau^{\prime}}\right) \geq \bmod \left(\Omega_{\tau}\right)$ and $\bmod \left(\Omega_{\tau^{\prime}}\left(\alpha^{\prime}\right)\right)$ decreases continuously to 0 as $\alpha^{\prime} \rightarrow 0$. Consequently, there is a unique value of $\alpha^{\prime}>0$ for which $\bmod \left(\Omega_{\tau^{\prime}}\left(\alpha^{\prime}\right)\right)=\bmod \left(\Omega_{\tau}\right)$.

Since the moduli are equal, there is a conformal map $\tilde{f}: \Omega_{\tau} \rightarrow \Omega_{\tau^{\prime}}\left(\alpha^{\prime}\right)$. By Schwarz reflection, $\tilde{f}$ sends points on the edges of $\Omega_{\tau}$ which correspond under $\Gamma_{\tau}$ to points on the edges of $\Omega_{\tau^{\prime}}\left(\alpha^{\prime}\right)$ which correspond under $\Gamma_{\tau^{\prime}}$. Thus $\tilde{f}$ projects to a map $f: R_{\tau} \rightarrow R_{\tau^{\prime}}$ which is conformal off $\gamma$.

Now let $\widetilde{\varphi}_{\tau, \tau^{\prime}}=\widetilde{f}^{-1}(\widetilde{f}(z)+1)$ on $\gamma^{+}$. Then $\widetilde{\varphi}$ projects to a welding map $\varphi$ on $\gamma$. Notice that $f\left(z^{+}\right)=f \circ \varphi\left(z^{-}\right)$on $\gamma$. 
The case $\operatorname{Im} \tau^{\prime} \geq \operatorname{Im} \tau$ is similar. By bending a horizontal geodesic, we can increase the modulus of $\Omega_{\tau^{\prime}}$ until it equals that of $\Omega_{\tau}$. The existence of a welding then follows precisely as before.

It follows from the well-known behavior of conformal maps at corners that in shrinking the angle at $\tau$ and $0, \varphi$ must act locally at $\tau$ and 0 like a power map $z \mapsto z^{\beta}$, with $\beta<1$. As a result, 0 and $\tau$ are not only fixed points of $\varphi$, but attracting fixed points.

Conversely, at the midpoint of the side between 0 and $\tau$, the straight line is bent to form an angle measuring more than $\pi$. Thus $\varphi$ acts locally at the midpoint like a power map $z \mapsto z^{\beta}, \beta>1$. Hence, the midpoint is a repelling fixed point. See Figure 3.

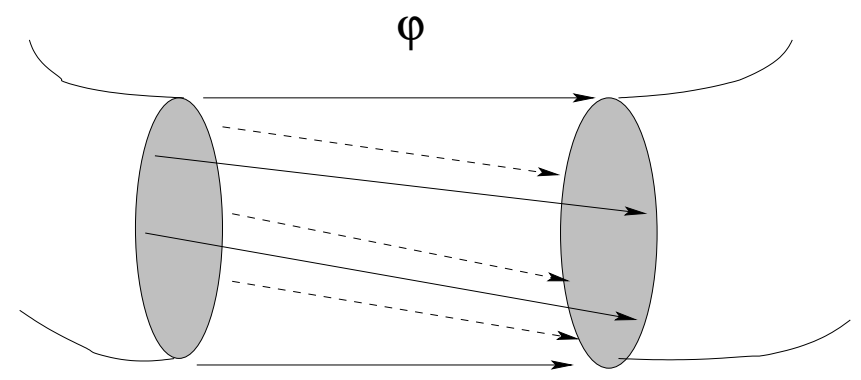

Figure 3. The welding map $\varphi$ constructed in the proof of Theorem 1.1 glues two points of $\gamma^{+}$back to their original positions and then "pulls" the remainder of $\gamma^{+}$away from one fixed point and toward the other.

Notice also that there was no great need to take $\alpha^{\prime} \leq \alpha_{0}$ in the above proof. There is also a unique $\alpha^{\prime} \geq \alpha_{0}$ so that $\bmod \left(\Omega_{\tau^{\prime}}\left(\alpha^{\prime}\right)\right)=\bmod \left(\Omega_{\tau}\right)$. This $\alpha^{\prime}$ will bend to the left; thus we obtain welding maps which stretch in the other direction.

3.2. Computing the welding map. The welding map $\varphi_{\tau, \tau^{\prime}}$ used in the proof of Theorem 1.1 can be expressed explicitly using Schwarz-Christoffel maps.

First observe that the unit disk $\mathbb{D}$ is taken onto the parallelogram $\Omega_{\tau}$ by the Schwarz-Christoffel map

$$
f_{1}(z)=c_{1} \int_{-1}^{z}(1-\xi)^{\alpha-1}\left(1-e^{-i \theta_{0}} \xi\right)^{-\alpha}\left(1+e^{i \theta_{0}} \xi\right)^{\alpha-1}(1-\xi)^{-\alpha} d \xi
$$

where $\alpha \pi=\pi-\operatorname{Arg}(\tau)$ is the exterior angle at $\tau+1$ and $c_{1}$ and $\theta_{0}$ are defined by the requirements that

$$
f(1)=c_{1} \int_{-1}^{1}(1-\xi)^{\alpha-1}\left(1-e^{-i \theta_{0}} \xi\right)^{-\alpha}\left(1+e^{i \theta_{0} \xi}\right)^{\alpha-1}(1-\xi)^{-\alpha} d \xi=1
$$



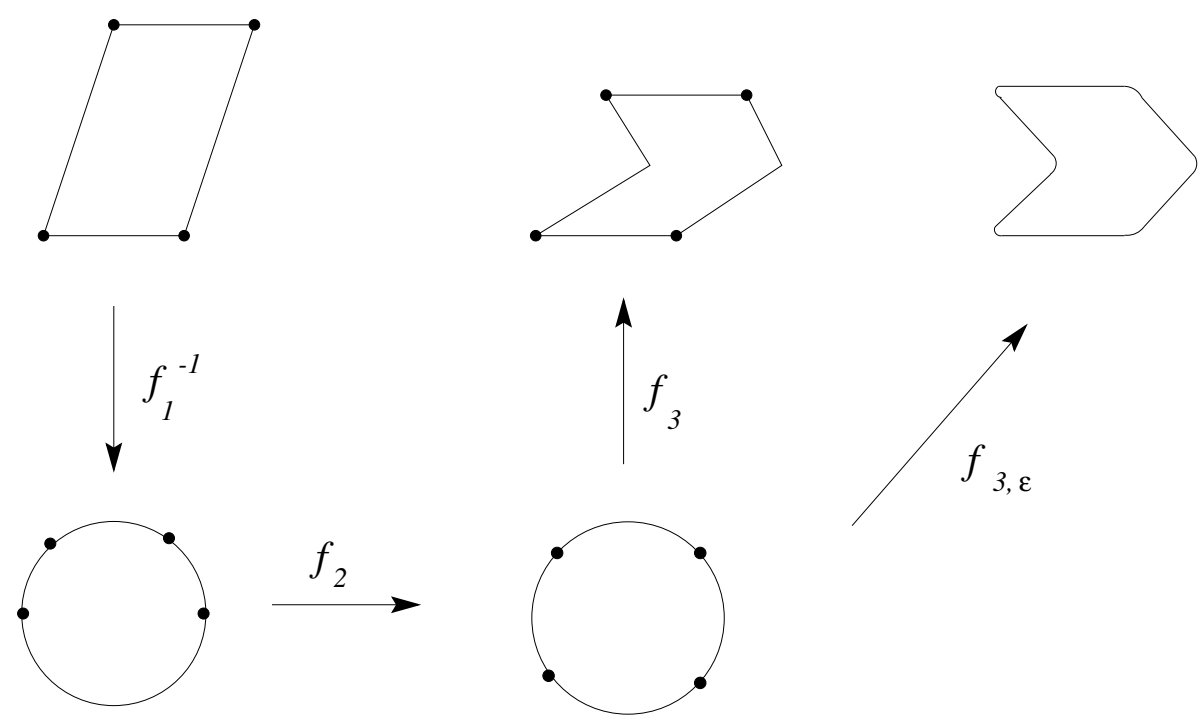

Figure 4. Our conformal welding is given by the composition $f_{3} f_{2} f_{1}^{-1}$. The images of the four corners of $\Omega_{\tau}$ are indicated by dots.

and $f_{1}\left(-e^{-i \theta_{0}}\right)=\tau$. See Figure 4 .

The map $f_{1}$ takes $-1,-e^{-i \theta_{0}}, e^{i \theta_{0}}$ and 1 onto the corners $0, \tau, 1+\tau$ and 1 , respectively. To take advantage of the symmetry of the region $\Omega_{\tau^{\prime}}\left(\alpha^{\prime}\right)$, we use the map

$$
f_{2}(z)=\frac{z-i r}{1+i r z}
$$

with $\frac{1-i r}{1+i r}=e^{-i \frac{\theta_{0}}{2}}$, to take $-1,-e^{-i \theta_{0}}, e^{i \theta_{0}}$ and 1 to the symmetric points $\pm e^{ \pm i \frac{\theta_{0}}{2}}$.

Now these points are mapped to the corners of $\Omega_{\tau^{\prime}}\left(\alpha^{\prime}\right)$ by the SchwarzChristoffel map

$$
\begin{aligned}
f_{3}(z)= & c_{3} \int_{-1}^{z}(1-\xi)^{1-2 \alpha^{\prime}}\left(1-2 \cos \left(\theta_{0} / 2\right) \xi+\xi^{2}\right)^{-\alpha^{\prime}} \\
& \left(1+2 \cos \left(\theta_{0} / 2\right) \xi+\xi^{2}\right)^{\alpha^{\prime}-1}(1+\xi)^{2 \alpha^{\prime}-1} d \xi+1-\frac{\overline{\tau^{\prime}}}{2},
\end{aligned}
$$

where $c_{3}$ is chosen so that $f_{3}\left(e^{i \theta_{0}}\right)=1+\tau^{\prime}$. Our desired welding map is then given by $\varphi_{\tau, \tau^{\prime}}=f_{3} f_{2} f_{1}^{-1}$ restricted to the left side of $\Omega_{\tau}$.

Notice, however, that the sharp corners of $\Omega_{\tau_{n}}$ destroy the smoothness of $f_{3}$ there. We can obtain a bilipschitz approximation to $f_{3} f_{2} f_{1}^{-1}$ if we replace $\Omega_{\tau^{\prime}}\left(\alpha^{\prime}\right)$ by a region $\widehat{\Omega}_{\tau^{\prime}}\left(\alpha^{\prime}\right)$ in which these corners have been smoothly 
rounded. This technique was used in $[\mathbf{3}]$. We replace $f_{3}$ by

$$
\begin{aligned}
f_{3, \epsilon}(z)=c_{4} \int_{-1}^{z} & {\left[\left(1-\xi e^{i \epsilon}\right)^{2 \alpha^{\prime}}+\left(1-\xi e^{-i \epsilon}\right)^{2 \alpha^{\prime}}\right] } \\
& {\left[\left(1-\xi e^{i\left(\theta_{0} / 2-\epsilon\right)}\right)^{1-\alpha^{\prime}}+\left(1-\xi e^{i\left(\theta_{0} / 2+\epsilon\right)}\right)^{1-\alpha^{\prime}}\right] } \\
& {\left[\left(1+\xi e^{i\left(-\theta_{0} / 2-\epsilon\right)}\right)^{1-\alpha^{\prime}}+\left(1+\xi e^{i\left(-\theta_{0} / 2+\epsilon\right)}\right)^{1-\alpha^{\prime}}\right] } \\
& {\left[\left(1+\xi e^{i\left(\theta_{0} / 2-\epsilon\right)}\right)^{\alpha^{\prime}}+\left(1+\xi e^{i\left(\theta_{0} / 2+\epsilon\right)}\right)^{\alpha^{\prime}}\right] } \\
& {\left[\left(1+\xi e^{i\left(-\theta_{0} / 2-\epsilon\right)}\right)^{\alpha^{\prime}}+\left(1+\xi e^{i\left(-\theta_{0} / 2+\epsilon\right)}\right)^{\alpha^{\prime}}\right] } \\
& {\left[\left(1-\xi e^{i \epsilon}\right)^{1-2 \alpha^{\prime}}+\left(1-\xi e^{-i \epsilon}\right)^{1-2 \alpha^{\prime}}\right] d \xi+1-\frac{\bar{\tau}}{2} }
\end{aligned}
$$

where $\epsilon$ is small and $c_{4}$ is chosen so that $f_{3, \epsilon}\left(e^{i \theta_{0} / 2}\right)=1+\tau^{\prime}$.

Thus we obtain a $C^{1}$ welding map $\varphi_{\tau, \tau^{\prime}, \epsilon}=f_{3, \epsilon} f_{2} f_{1}^{-1}$. The smoothness of $\varphi_{\tau, \tau^{\prime}}$ will prove useful in constructing a circle packing version of Theorem 1.1.

Remark 3.3. The reader may object to the description of $\varphi_{\tau, \tau^{\prime}}$ as "explicit" since an inverse for $f_{1}$ was not given in closed form. In practice, however, the inverse is obtainable by recently developed Schwarz-Christoffel packages to the desired accuracy $[\mathbf{9}]$.

Remark 3.4. The nature of conformal weldings of tori is in marked contrast to the well-studied problem of welding discs $[11,14,16,19,21,27$, 29]. Since there is only one possible conformal structure on the sphere formed by welding discs, welding discs does not alter position in moduli space. Moreover, except for a few special cases, it has not been possible to find either a closed form of the conformal welding or an explicit description of the "seam" resulting from a given welding. The best known result is the discrete approximation scheme developed in [27].

\section{Circle packing.}

4.1. Abstract triangulations. We will use the term abstract triangulation to mean a simplicial 2-complex. In particular, these are purely combinatorial objects. They possess no geometric structure until they are embedded in a surface.

We also require our abstract triangulations to be CP-complexes; however, notice that the restrictions imposed by Conditions (2) through (4) are extremely mild and are met by any reasonable complex.

Definition 4.1. A CP-complex $\mathcal{K}$ is an abstract simplicial 2-complex such that:

(1) $\mathcal{K}$ is simplicially equivalent to a triangulation of an (orientable) surface.

(2) Every boundary vertex of $\mathcal{K}$ has an interior neighbor.

(3) The collection of interior vertices is nonempty and edge-connected. 
(4) There is an upper bound on the degree of vertices in $\mathcal{K}$, that is, on the number of edges incident at each vertex.

\subsection{Circle packings.}

Definition 4.2. A circle packing is a configuration of circles with a specified pattern of tangencies. In particular, if $\mathcal{K}$ is a $\mathrm{CP}$-complex, then a circle packing $P$ for $\mathcal{K}$ is a configuration of circles such that:

(1) $P$ contains a circle $\mathcal{C}_{v}$ for each vertex $v$ in $\mathcal{K}$,

(2) $\mathcal{C}_{v}$ is externally tangent to $\mathcal{C}_{u}$ if $[v, u]$ is an edge of $\mathcal{K}$,

(3) $\left\langle\mathcal{C}_{v}, \mathcal{C}_{u}, \mathcal{C}_{w}\right\rangle$ forms a positively oriented mutually tangent triple of circles if $\langle v, u, w\rangle$ is a positively oriented face of $\mathcal{K}$.

A packing is called univalent if none of its circles overlap, that is, if no pair of circles intersect in more than one point.

A univalent circle packing produces a geometric realization of its underlying complex. Vertices can be embedded as centers of their corresponding circles, and edges can be embedded as geodesic segments joining centers of circles. This embedding for $\mathcal{K}$ is called the carrier of the packing, and denoted by $|K|$.

If $\mathcal{K}$ is embedded in $\mathbb{C}$ in two different ways, there is a natural piecewise affine map sending triangles in one embedding to triangles in the other. If the embeddings for $\mathcal{K}$ are created using circle packings, we will refer to the induced map as a discrete conformal map. As the name suggests, there are deep connections between discrete conformal maps and their classical counterparts.

4.3. Packable surfaces. Various existence results for circle packings have been given by Thurston [24], Minda and Rodin [17], and Beardon and Stephenson [4]. Moreover, Brooks [8] showed that compact packable surfaces are dense in moduli space. His result was extended to surfaces of finite analytic type by Bowers and Stephenson $[\mathbf{6}, 7]$. In summary:

Theorem 4.3. Let $\mathcal{K}$ be an abstract triangulation of a surface $R$ of finite analytic type. Then there is a unique point in the moduli space for $R$ which supports a packing for $\mathcal{K}$. Similarly, $\mathcal{K}$ and a marking for $R$ determine a unique packable point in the Teichmüller space of $R$. Moreover, the collection of all packable surfaces is dense in both the moduli space and Teichmüller space of $R$.

Unfortunately, a thorough description of the effect of $\mathcal{K}$ on the global geometry of $R$ has been extremely elusive. For hyperbolic surfaces, the effect of combinatorial manipulation of $\mathcal{K}$ by earthquakes has been studied in [28]. In Section 6, we will consider combinatorial deformations of tori analogous to the conformal weldings of Section 3.1. 
4.4. Combinatorial influence on geometry. It has been known for some time that the local combinatorics of a packing exert an important influence on the local geometry. Rodin and Sullivan's Ring Lemma [22] connects the combinatorial properties of packings and the function theoretic properties of associated discrete conformal maps.

Ring Lemma. Suppose $\mathcal{K}$ is a complex of degree $D$. If $v$ an interior vertex of $\mathcal{K}$ and $w$ is a neighbor of $v$, then there is a lower bound $C_{D}$ on the ratio of the radius of $\mathcal{C}_{w}$ to $\mathcal{C}_{v}$ in any univalent packing for $\mathcal{K}$. In particular, the lengths of any two sides of a triangle in $|\mathcal{K}|$ are $C_{D}$-comparable.

The constant $C_{D}$ of the Ring Lemma has been computed by Aharonov [1, 2] to be

$$
C_{D}=\frac{1}{a_{D-2}^{2}+a_{D-1}^{2}-1},
$$

where $a_{i}$ is the $i^{t h}$ Fibonacci number. This bound is sharp.

It is easy to show that affine maps between triangles are quasiconformal with dilatation depending only on the difference in corresponding angles of the triangles. The Ring Lemma implies that angles at interior vertices in the carrier of a univalent packing are bounded away from 0 and $\pi$. This gives an upper bound on the difference between corresponding angles in the carriers of two different packings for the same complex. Consequently, discrete analytic functions are $k$-quasiconformal on faces which do not contain a boundary vertex, where $k$ depends only on the degree of the packing.

Even greater control on the dilatation is possible by considering more generations of circles. The resulting Packing Lemma was first shown for packings in which every vertex has degree $6[\mathbf{2 2}, \mathbf{2 5}]$ and later extended by Stephenson [23] and He and Rodin [12].

Definition 4.4. A chain of circles in packing $P$ for $\mathcal{K}$ is a collection of circles $\mathcal{C}_{v_{1}}, \mathcal{C}_{v_{2}}, \ldots, \mathcal{C}_{v_{n}}$ of $P$ so that $v_{i}$ and $v_{i+1}$ share an edge in $\mathcal{K}, i=$ $1,2, \ldots, n-1$, and $v_{i} \neq v_{j}$, if $i \neq j$. Thus a chain describes a non-selfintersecting edge path in $\mathcal{K}$. A chain is closed if $v_{1}=v_{n}$.

Packing Lemma. If a circle $\mathcal{C}_{v}$ of a circle packing $P$ is surrounded by $n$ closed chains of circles, each circle having degree at most $m$, then the dilation of a discrete conformal map defined on faces containing $v$ decreases to 0 as $n \rightarrow \infty$.

Finally the Length-Area Lemma [22] provides combinatorial control over the size of circles. It is a type of combinatorial isoperimetric inequality.

Length-Area Lemma. Let $P$ be a univalent circle packing in $\mathbb{D}$ and $\mathcal{C}_{v}$ a circle in $P$ with euclidean radius $r$. Assume there are $m$ disjoint chains of 
circles in $P$ having combinatorial lengths $n_{1}, n_{2}, \ldots, n_{m}$, and each separating $\mathcal{C}_{v}$ from the origin and a point on the boundary. Then

$$
r \leq \frac{1}{\sqrt{\sum_{i=1}^{m} \frac{1}{n_{i}}}} .
$$

\section{Simple combinatorial deformations.}

5.1. Changing the triangulation vs. changing the embedding. Recall that our abstract triangulations are purely combinatorial objects until they are embedded in some surface, usually by means of a circle packing. If a triangulation $\mathcal{K}$ is realized by a packing of a surface $\tau$, then changing $\mathcal{K}$ will lead not only to a new complex $\mathcal{K}^{\prime}$, but $\mathcal{K}^{\prime}$ will also induce a new packing on a (most likely different) surface $\tau^{\prime}$.

If we wish to directly compare the circle packing-induced geometries of $\tau$ and $\tau^{\prime}$ we must limit the type of changes we make to $\mathcal{K}$. In particular, any combinatorial change we make to $\mathcal{K}$ should be realizable in $\tau$ by adding or moving edges of $|\mathcal{K}|$. These in situ changes to $|\mathcal{K}|$ will produce an embedding of the new complex $\mathcal{K}^{\prime}$ in $\tau$. Now we construct a discrete conformal map $f$ sending triangles in the embedding of $\mathcal{K}^{\prime}$ in $\tau$ to the corresponding triangles in the embedding of $\mathcal{K}^{\prime}$ in $\tau^{\prime}$. By thus comparing the embeddings of $K^{\prime}$ in $\tau$ and in $\tau^{\prime}$, we can compare the geometry of $\tau$ and $\tau^{\prime}$.

If the in situ modification to $|\mathcal{K}|$ can be made in such a way that the angles in the newly embedded triangles are bounded away from 0 and $\pi$ and if the degree of $\mathcal{K}$ is controlled, then using the Ring Lemma, the dilatation of $f$ can be estimated and an upper bound on the Teichmüller distance between $\tau$ and $\tau^{\prime}$ computed.

For the remainder of the this section, we will consider examples of these combinatorial modifications which

1) permit bounds on the degree of the new complex

2) can be realized by an in situ deformation of the original embedding while maintaining control over angles.

5.2. Hex refinement. A particularly useful example of this type of combinatorial deformation is the hex refinement scheme of Bowers and Stephenson $[5]$.

Definition 5.1. The hex refinement of a complex $\mathcal{K}$ is the complex formed by adding a vertex to each edge and adding an edge between new vertices lying on the same face. See Figure 5.

Notice that any new interior vertices added to $\mathcal{K}$ by hex refinement have degree 6 , while the degrees of the original vertices remain unchanged.

Aside from the embedding provided by circle packing, there is another useful embedding of the hex refinement of $\mathcal{K}$. If $\mathcal{K}$ is embedded in $\mathbb{C}$ in 

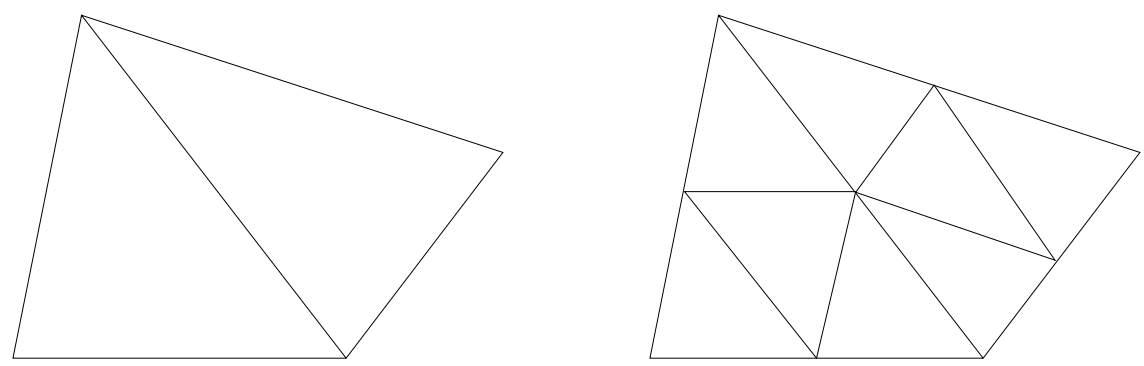

Figure 5. Two triangles before (left) and after (right) hex refinement. Notice that the new interior vertex has degree 6 .

such a way that edges correspond to euclidean line segments, then its hex refinement may be realized by adding line segments joining the mid-point of each edge. In this case, each face of $\mathcal{K}$ will be subdivided into four new faces, each similar to the original and having edges one-half as long.

This second embedding is generally different from the embedding provided by circle packing. Since the faces are broken into similar faces, no new geometry emerges. By contrast, the circle packings can make fine adjustments to the embeddings as $\mathcal{K}$ is repeatedly refined. In fact, the Packing Lemma implies that after many hex refinements, most triangles embedded by a circle packing are nearly equilateral. This phenomenon leads to the following result of Bowers and Stephenson [5].

Lemma 5.2. Let $\mathcal{K}$ be an abstract triangulation of a compact Riemann surface realized by a packing $P_{n}$ on a surface $R_{\tau_{n}}$ and suppose $\mathcal{K}_{n}$ is the $n^{\text {th }}$ hex refinement of $\mathcal{K}$. Then $\left\{R_{\tau_{n}}\right\}$ converges in the Teichmüller metric to the point $\tau_{\infty}$ corresponding to the surface formed by gluing together equilateral triangles in the pattern given by $\mathcal{K}$. In particular, $\tau_{\infty}$ lies in a ball centered at $\tau$ with radius determined only by the degree of $\mathcal{K}$.

5.3. Trivalent barycentric subdivision. A second very instructive example is provided by trivalent barycentric subdivision, or starring from the barycenter. In this refinement scheme, a face is subdivided by adding a vertex with edges to each of the three existing vertices. See Figure 6 .

Proposition 5.3. Trivalent barycentric subdivision doubles the degree of every interior vertex. Notice that the new complex can be embedded using the original packing by adding a vertex at the barycenter of each face and embedding the new edges as geodesic segments.

Since the degree of the complex grows without bound, the Ring Lemma and Packing Lemma are useless to control the angles in the refined complex (and hence the distortion of discrete maps). 

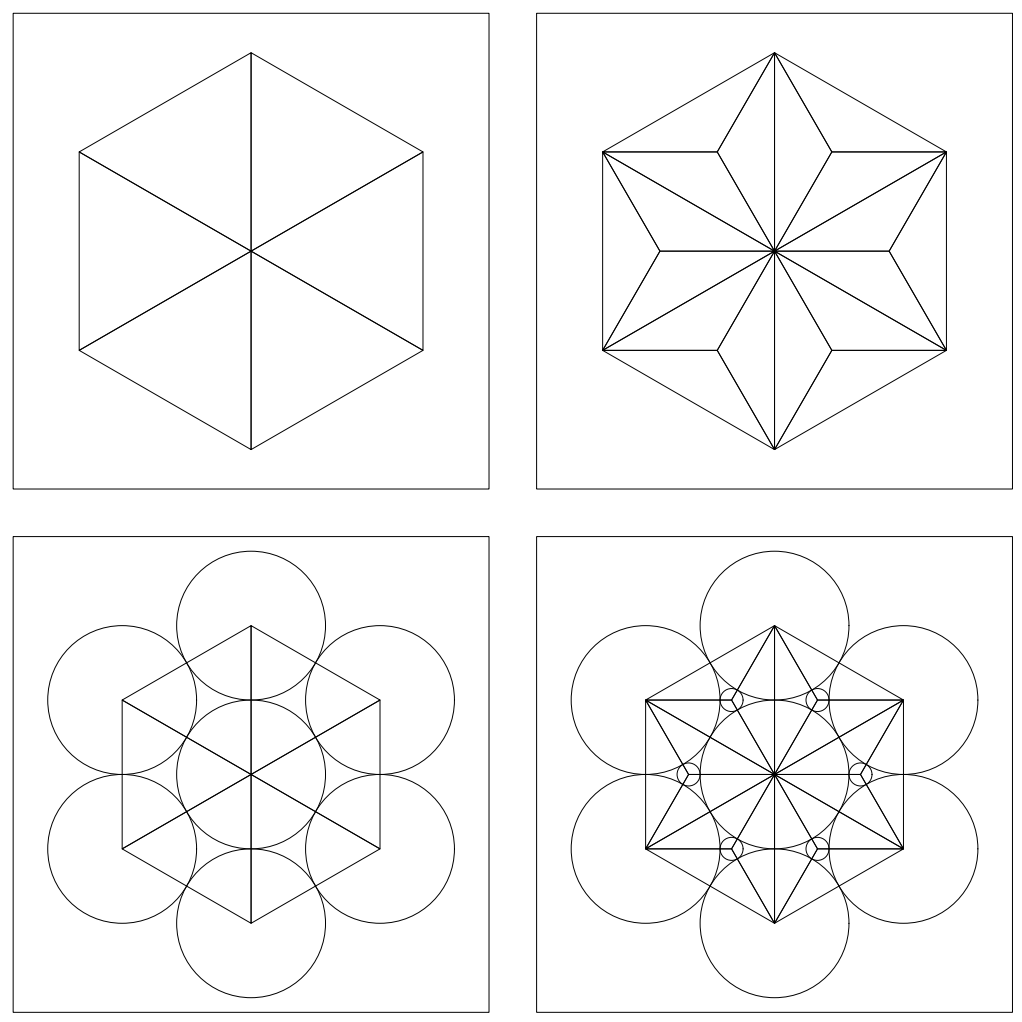

Figure 6. The effect of trivalent barycentric subdivision on a complex (top left) and its packing (lower left). Notice the subdivided complex (top right) has changed, but the six large circles in the new packing (lower right) are unchanged.

Surprisingly, however, trivalent barycentric subdivision does not affect the location of the packed surface in moduli space at all! A packing for this complex is formed by simply adding a circle to each interstice without moving any of the original circles.

5.4. Vertex-edge subdivision. Notice that if a vertex is added to an edge of an existing triangle, this triangle will become a quadrilateral, having four vertices instead of only three. Thus without additional modifications, our complex will cease to be a triangulation.

This situation can be easily remedied by adding a new edge to the complex. See Figure 7. The new edge can obviously be embedded in $|\mathcal{K}|$ as a line segment. It was shown in [27] that if the new vertex is bounded away from the old vertices, the angles created by this vertex-edge subdivision will be bounded. 


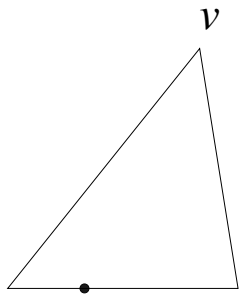

w

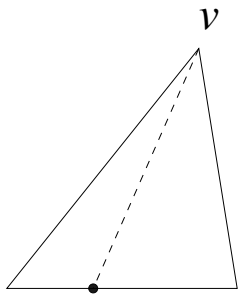

w

Figure 7. The addition of a vertex $w$ to an edge (left) can be embedded by simply adding a new edge $[v, w]$ (right).

Lemma 5.4. If a vertex $w$ is added to an edge $e$ of length $l$ and the distance from $w$ to the endpoints of $e$ is at least $\epsilon l$, then the angles created by vertexedge subdivision are bounded away from 0 and $\pi$ by a constant depending only on $\epsilon$.

5.5. Local hex refinement. A final example is provided by "local hex refinement". See Figure 8. Notice that the hex refinement procedure described in Section 5.2 adds a vertex to each edge of the complex. If we wish to refine only one triangle, we quickly run into difficulty since adding a vertex to an interior edge affects the neighboring triangle as well.

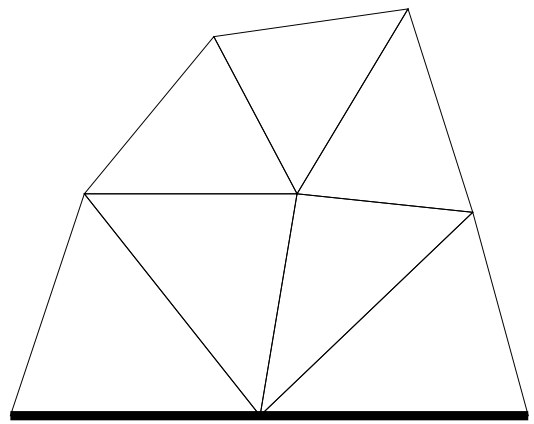

$\gamma$

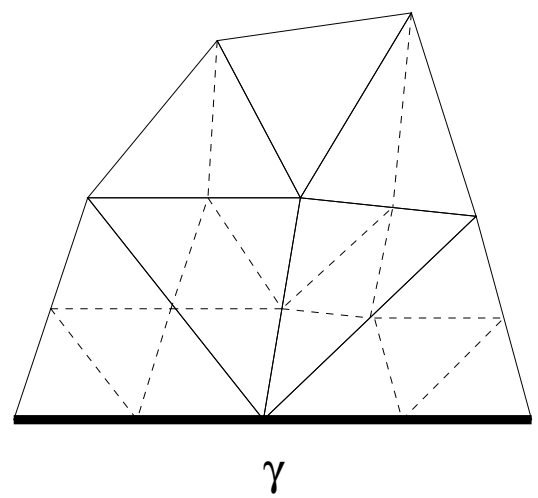

$\gamma$

Figure 8. A complex (left) can be locally hex refined along an edge path $\gamma$ (right).

It is possible, however, to refine in a neighborhood of an edge path $\gamma$ without affecting the remainder of the complex. Let $G$ be the collection of triangles hitting $\gamma$. We first hex refine triangles in $G$ as before. Notice, however, that this procedure adds a vertex to the midpoint of all triangles 
which share an edge with $G$. We cannot hex refine these neighboring triangles, as that would force us to propagate the refinement throughout the entire complex. Instead, we use vertex-edge subdivision to absorb these extra vertices.

Notice that all angles created by hex refinement have the same bounds as the original angles. Similarly, the angles created by vertex-edge subdivision are bounded by Lemma 5.4. Finally, notice that the degree of the locally hex refined complex is bounded by the larger of 6 and $D+1$, where $D$ is the original degree of $\mathcal{K}$.

\section{Combinatorial welding deformations.}

6.1. Correctly cutting a torus. We now describe a combinatorial deformation analogous to the conformal welding process. Suppose $\mathcal{K}$ is an abstract triangulation of a torus with degree $D$. By Theorem 4.3 (or originally, [4]), there is a circle packing $P_{\tau}$ on a torus $R_{\tau}$. We would like to cut $\mathcal{K}$ along some geodesic $\gamma$ and reattach the two copies $\gamma^{+}$and $\gamma^{-}$of $\gamma$ using a welding map $\varphi: \gamma^{+} \rightarrow \gamma^{-}$.

Unfortunately, the $\gamma$ is an object from the continuous world, not one of our discrete creations. In general, $\gamma$ will pass indiscriminately through triangles and vertices of $|\mathcal{K}|$ with no regard for the underlying combinatorial structure. Thus we need to construct an edge path of $\mathcal{K}$ corresponding to $\gamma$.

One option is to completely replace $\gamma$ with a polygonal edge path embedded nearby. This approach is used, for example, in [28] to cut open hyperbolic triangulations and approximate earthquakes. We prefer a different construction here, adding edges to $|\mathcal{K}|$ so that $\gamma$ itself becomes the desired edge path.

If some portions of $\gamma$ already correspond to edges of $|\mathcal{K}|$, then we accept these edges without modification. Elsewhere, however, as $\gamma$ enters each triangle $T$, it joins either a vertex to an edge or, more commonly, an edge to an edge.

In the first case, $\gamma$ splits $T$ into two smaller triangles. Thus it would be combinatorially acceptable to merely add a new edge to $\mathcal{K}$ corresponding to this segment of $\gamma$. Unfortunately, we must embed the new edge using this segment of $\gamma$ since we need our welding map $\varphi: \gamma^{+} \rightarrow \gamma^{-}$to act on our new edge path. This addition might unacceptably alter the embedding $|\mathcal{K}|$. In particular, if $\gamma$ exits $T$ very near an existing vertex $v_{1}$, one of the new triangles will have a small angle. See Figure 9.

To avoid this difficulty, we will slightly alter our embedding $|\mathcal{K}|$ by moving $v_{1}$ to lie on $\gamma$. We let $\epsilon=\frac{1}{3 C_{D}}$, where $C_{D}$ is the constant from the Ring Lemma. If the distance from $v_{1}$ to $\gamma$ is less than $\epsilon$ times the minimum side length of $T$, then we will move $v_{1}$ to the closest point on $\gamma$. Notice that the angles in a euclidean triangle vary continuously with the side lengths, and 


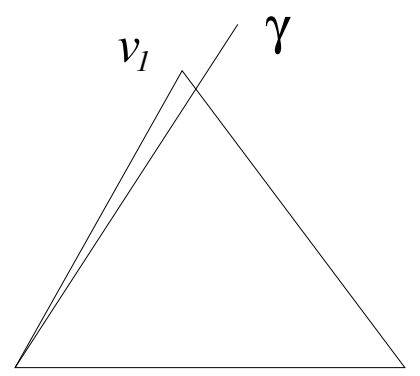

Figure 9. If $\gamma$ hits one vertex and passes very near another, the resulting angle will be small.

all the triangles in $|\mathcal{K}|$ containing $v_{1}$ have $C_{D^{-c o m p a r a b l e}}$ side lengths by the Ring Lemma. Thus we maintain a uniform (albeit weaker) bound on the angles.

In the second case, if the distance from $\gamma$ to a vertex is less than $\epsilon$ times the minimum side length of $T$, we again move that vertex to $\gamma$. Thus we may assume that $\gamma$ is bounded away from the vertices of $T$. Notice that adding an edge corresponding to $\gamma \cap T$ divides $T$ into a smaller triangle $T_{1}$ and a quadrilateral. We then add another edge to divide the quadrilateral into triangles $T_{2}$ and $T_{3}$. See Figure 10 .

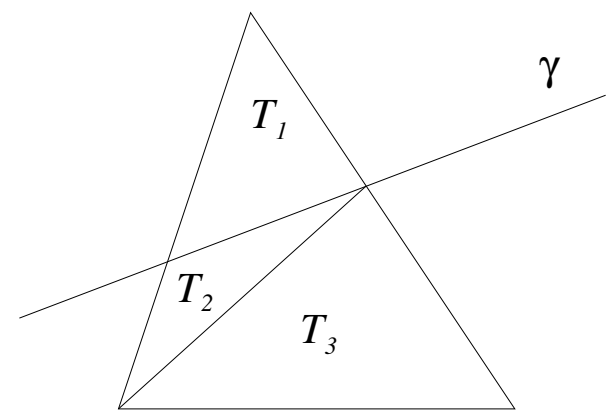

Figure 10. Adding an edge corresponding to $\gamma$, we form a triangle $T_{1}$ and a quadrilateral. A second edge then breaks the quadrilateral into triangles $T_{2}$ and $T_{3}$.

Lemma 6.1. All the angles in $T_{1}, T_{2}$, and $T_{3}$ are bounded away from 0 and $\pi$. This bound depends only on the degree of $\mathcal{K}$.

The proof involves only elementary geometry and is similar to the proof of Lemma 5.4 found in [27].

6.2. Combinatorial re-attachment. Once our torus has been cut open, we have two copies of the edge path $\gamma$, one of which we label $\gamma^{+}$and the 
other which we label $\gamma^{-}$. If $\varphi: \gamma^{+} \rightarrow \gamma^{-}$is an orientation reversing homeomorphism, then we can re-attach these two copies using $\varphi$ as our guide. A general procedure for performing this re-attachment is described in [28]; however, for completeness, we will briefly recount it here.

If $\varphi$ maps each edge of $\gamma^{+}$to a corresponding edge in $\gamma^{-}$, then we merely glue the edges together to form a new abstract triangulation. However, most welding maps - and certainly those of the type described in Section 3.2 - will not respect the combinatorial structures of $\gamma^{+}$and $\gamma^{-}$in this way. Thus it is necessary to modify $\mathcal{K}$ so that $\varphi$ will respect the modified combinatorics.

A first attempt at a solution might be:

(1) For each $v \in \gamma^{+}$, add a vertex to $\gamma^{-}$corresponding to $\varphi(v)$.

(2) For each $w \in \gamma^{-}$, add a vertex to $\gamma^{+}$corresponding to $\varphi^{-1}(w)$.

Now $\varphi$ will respect the combinatorics of $\gamma^{+}$and $\gamma^{-}$.

Of course, we cannot simply add vertices to $\mathcal{K}$ and expect that $\mathcal{K}$ will remain a triangulation. Each triangle to which a new vertex was added must be subdivided in some way. The vertex-edge subdivision described in Section 5.4 is precisely the tool we need.

However, since we have a future application of the Ring Lemma in mind, our modifications must maintain a uniform bound on the degree of $\mathcal{K}$ and on the angles in any new triangles we create. Lemma 5.4 allows us to control the angles created by the vertex-edge subdivision provided the new vertices are not placed too near either each other or the existing vertices.

A priori, $\varphi$ might map vertices of $\gamma^{+}$all over the place, defying any attempts to control angles or the degree of $\mathcal{K}$. However, if $\varphi$ is bilipschitz and the lengths of edges of $\gamma^{+}$and $\gamma^{-}$are uniformly comparable, then it is a relatively simple matter to find such bounds [27].

Thus we will hereafter assume that $\varphi$ is $B$-bilipschitz; that is, for all $x, y \in \gamma^{+}$,

$$
\frac{1}{B}|x-y|_{\gamma^{+}}<|\varphi(x)-\varphi(y)|_{\gamma^{-}}<B|x-y|_{\gamma^{+}},
$$

where $|x-y|_{\gamma^{+}}$denotes the distance from $x$ to $y$ inside $\gamma^{+}$and $|\varphi(x)-\varphi(y)|_{\gamma^{-}}$ denotes the distance from $\varphi(x)$ to $\varphi(y)$ inside $\gamma^{-}$. Since bilipschitz maps are quasisymmetric, Theorem 3.2 will hold.

It follows from the Ring Lemma and the control on our modifications that the lengths of adjacent edges of $\gamma^{+}$and $\gamma^{-}$are $C_{D^{-}}$-comparable. Thus edges which are separated by $n$ vertices are $C_{D}^{n}$-comparable. Unfortunately, since this bound grows with $n$, if we attempt to weld a sequence of finer and finer complexes (as we will in Section 7), we cannot maintain uniform control throughout the entire sequence. We need a bound which depends only on the degree of $\mathcal{K}$ and not on the number of edges in $\mathcal{K}$.

Our remedy is to break edges which are too long into shorter pieces using the local hex refinement scheme of Section 5.5. More precisely, let $r$ be the 
minimum edge length of $\gamma^{+} \cup \gamma^{-}$. Let $E_{1}$ be the set of all edges in $\gamma^{+} \cup \gamma^{-}$ with length greater than $C_{D} r$.

Recall that our method for embedding a local hex refinement along an edge path splits edges in the path in half. Thus we can locally hex refine each component of $E_{1}$ and reduce the edge lengths by a factor of 2 . Recall that edges of $\gamma^{+} \cup \gamma^{-}$which share a vertex with an edge of $E_{1}$ will also be split in half.

Now let $E_{2}$ be the set of all edges in $\gamma^{+} \cup \gamma^{-}$whose length is still greater than $C_{D} r$. Local hex refinement along $E_{2}$ will again reduce edge lengths by a factor of 2 . Repeating this process, we see that after finitely many steps all the edges in $\gamma^{+} \cup \gamma^{-}$will have length at most $C_{D} r$.

Moreover, any edge which is created at step $n$ but is not split at step $n+1$ will have length at least $\frac{1}{2} C_{D} r$; otherwise, it would have been too short to be split at stage $n$. This does not yet give a lower bound on the lengths of all edges after all the splitting, since it is possible such an edge might be split once more (and only once more, by the Ring Lemma) if it shares a vertex with an edge which is in $E_{n+1}$. Thus the length $e$ of any edge formed by splitting an edge in some $E_{i}$ must satisfy

$$
\frac{1}{4 C_{D}} r<\frac{1}{4} C_{D} r \leq e \leq C_{D} r<4 C_{D} r .
$$

Of course, the length of any other edge must also satisfy (6.2) since it would have been too short to split. Thus after the splitting, all edges of

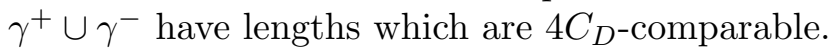

6.3. Properties of the combinatorial welding. Our modifications of the previous section now lead to a bound on the number of vertices which can be mapped by $\varphi$ or $\varphi^{-1}$ to any given edge.

Lemma 6.2. Adding vertices to $\gamma^{+} \cup \gamma^{-}$as described in (1) and (2) above will increase the degree of $\mathcal{K}$ by at most $4 C_{D} B$, where $D$ is the degree of $\mathcal{K}$ and $B$ is the bilipschitz constant of $\varphi$.

Proof. If $\left[w_{1}, w_{2}\right]$ is an edge of $\gamma^{-}$of length $e$, then by (6.1),

$$
\varphi^{-1}\left(\left[w_{1}, w_{2}\right]\right)<B e .
$$

But since edges of $\gamma^{+}$and $\gamma^{-}$are $4 C_{D^{-}}$comparable, at most $4 C_{D} B$ edges of $\gamma^{+}$can lie within a region of length $B e$. Thus at most $4 C_{D} B$ vertices of $\gamma^{+}$ can be mapped onto $\left[w_{1}, w_{2}\right]$.

The proof for edges of $\gamma^{+}$is similar.

In the same way, notice that $\varphi$ can map an edge of $\gamma^{+}$onto a region no smaller than $\frac{1}{4 C_{D} B}$ times the length of the shortest edge of $\gamma^{-}$. Thus the images of vertices cannot be too close together, and Lemma 5.4 ensures that the angles formed by our vertex-edge subdivision are bounded away from 0 and $\pi$ by a constant depending only on $B$ and $C_{D}$. 
The final concern is the possibility that new vertices will be added arbitrarily close to an existing vertex. If $\left[w_{1}, w_{2}\right]$ is an edge of length $e$ in $\gamma^{-}$, we define a "buffer zone" of length $\frac{1}{5 C_{D} B} e$ around $w_{1}$ and $w_{2}$. If $\varphi$ maps a vertex $v$ of $\gamma^{+}$into one of the buffer zones, we redefine $\varphi(v)$ and "round off" to the nearest vertex $w_{1}$ or $w_{2}$. Since $\varphi$ is $B$-bilipschitz and all edges have length at least $\frac{1}{4 C_{D}} e$, at most one vertex can be mapped into each buffer zone. Thus $\varphi$ is still a bilipschitz homeomorphism.

With no vertices added too closely to any existing vertices, Lemma 5.4 now implies that all angles in the in situ embedding for our newly augmented $\mathcal{K}$ are bounded.

\section{A discrete mode of transportation.}

Now we give a discrete proof of Theorem 1.1. This will enable us to move about in moduli space by prescribing combinatorial changes.

7.1. The starting point. Suppose $\mathcal{K}$ is an abstract triangulation of a torus with degree $D$. By Theorem $4.3, \mathcal{K}$ has a packing $P$ on a torus $R_{\tau}$. Choose $\tau^{\prime} \in \mathbb{H}$. As in the proof of Theorem 1.1, we will consider only the case $\tau^{\prime} \in\{z \mid 0<\operatorname{Im} z \leq \operatorname{Im} \tau\}$ and weld $R_{\tau}$ along a vertical geodesic. The case $\operatorname{Im} \tau^{\prime} \geq \operatorname{Im} \tau$ requires welding along a horizontal geodesic, but is in all other aspects identical.

Let $\mathcal{K}_{n}$ be the $n^{\text {th }}$ hex refinement of $\mathcal{K}$. Each $\mathcal{K}_{n}$ has a packing $P_{n}$ on some torus $R_{\tau_{n}}$. As discussed in Section 5.2, we would not expect $\tau_{n}$ to equal $\tau$, but by Lemma 5.2, $\left\{\tau_{n}\right\}$ will converge to a point $\tau_{\infty}$ near $\tau[5]$.

Our repeated combinatorial refinements will carry finer and finer geometric information when realized by circle packings. Indeed, a standard application of the Length Area Lemma [22] shows that the mesh of $P_{n}$ decreases to 0 as $n \rightarrow \infty$.

Fix a vertex $v \in \mathcal{K}$. Notice that a copy of $v$ remains in each refinement $\mathcal{K}_{n}$. Let $\gamma_{n}$ be the vertical geodesic passing through $v$. Cutting $R_{\tau_{n}}$ open along $\gamma_{n}$ yields two copies of $\gamma_{n}$, one of which we label $\gamma_{n}^{+}$and the other $\gamma_{n}^{-}$.

7.2. Directions for deforming the tori. In Section 3.2, we computed a useful form for the identification map $\varphi_{\tau_{\infty}, \tau^{\prime}}$ which welds $R_{\tau_{\infty}}$ into $R_{\tau^{\prime}}$. By rounding the corners of our fundamental region, we also obtained a $C^{1}$ approximation $\varphi_{\tau_{\infty}, \tau^{\prime}}^{\epsilon}$ to $\varphi_{\tau_{\infty}, \tau^{\prime}}$. Since $\varphi_{\tau_{\infty}, \tau^{\prime}}^{\epsilon}$ satisfies our bilipschitz requirement, we will use it to weld $\gamma_{n}^{+}$to $\gamma_{n}^{-}$. We will let $\epsilon \rightarrow 0$ and obtain a welding for $\varphi_{\tau_{\infty}, \tau^{\prime}}$ by a diagonalization argument.

Notice also that our hex refinements resulted in a sequence of surfaces converging to $R_{\tau_{\infty}}$, not the surface $\phi_{\tau_{\infty}}$ itself. Thus we must transfer the map $\varphi_{\tau_{\infty}, \tau^{\prime}}^{\epsilon}$ to each of the surfaces $R_{\tau_{n}}$.

Recall from Section 2, there is a map $M_{n}: R_{\tau_{n}} \rightarrow R_{\tau_{\infty}}$ having minimal dilatation. In fact, this Teichmüller map is just the projection to $R_{\tau_{n}}$ of a 
linear map of $\mathbb{R}^{2}$ which sends the basis $\left\{(1,0),\left(\operatorname{Re} \tau_{n}, \operatorname{Im} \tau_{n}\right)\right\}$ to the basis $\left\{(1,0),\left(\operatorname{Re} \tau_{\infty}, \operatorname{Im} \tau_{\infty}\right)\right\}$. In particular, $M_{n}$ is uniformly bilipschitz, and as $n \rightarrow \infty, M_{n}$ converges to the identity map.

Now the map

$$
M_{n}^{-1} \varphi_{\tau_{\infty}, \tau^{\prime}}^{\epsilon} M_{n}
$$

is an orientation-reversing homeomorphism from $\gamma_{n}^{+}$to $\gamma_{n}^{-}$. Moreover, we may assume the vertex $v \in \gamma_{n}$ corresponds to the fixed point of $M_{n}^{-1} \varphi_{\tau_{\infty}, \tau^{\prime}}^{\epsilon} M_{n}$.

We can use

$$
M_{n}^{-1} \varphi_{\tau_{\infty}, \tau^{\prime}}^{\epsilon} M_{n}
$$

to combinatorially deform $\mathcal{K}_{n}$ as described in Section 6 .

Qualitatively, this combinatorial welding holds $v$ fixed, pulls the remaining vertices away from $v$, and then glues the stretched path $\gamma_{n}^{+}$onto the unstretched $\gamma_{n}^{-}$. Finally, the resulting welded complexes $\mathcal{K}_{n}^{\prime}$ can be realized by circle packings $P_{n}^{\prime}$ on some tori $R_{\tau_{n}^{\prime}}$.

7.3. An example. Given the large amount of notation needed to describe the welding process, pausing for an example is certainly in order. Consider the circle packing on the torus $R_{\tau}$ depicted in Figure 11. Lifting to $\mathbb{H}$ and normalizing, it is easy to compute that $\tau$ is approximately $0.5832+0.866025 i$.

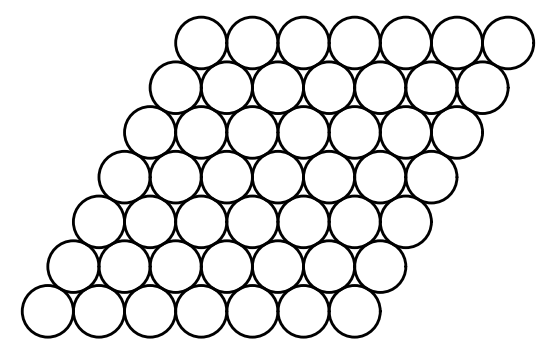

Figure 11. A packing on the torus $R_{0.5832+0.866025 i}$. The left and right sides are identified, as are the top and bottom.

Now consider the "square" torus $R_{\tau^{\prime}}$, with $\tau^{\prime}=i$. Our combinatorial welding proceedure yields the welded packing of Figure 12. The right and left sides of this packing correspond to the welding curve. 


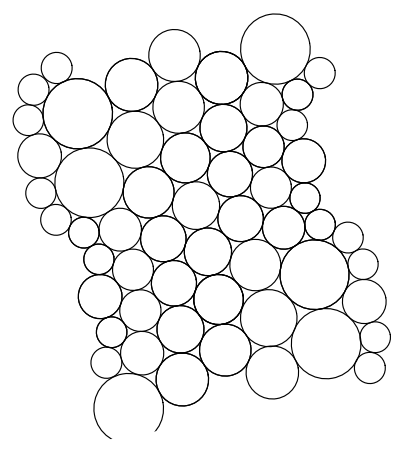

Figure 12. The torus of Figure 11 after welding.

7.4. Maps for our journey. Notice that except for the "seams" $\gamma_{n}$ and $\gamma_{n}^{\prime}$, the complexes $\mathcal{K}_{n}$ and the welded complexes $\mathcal{K}_{n}^{\prime}$ are combinatorially equivalent. Thus off $\gamma_{n}$ we can define a discrete conformal map

$$
f_{n}:\left|\mathcal{K}_{n}\right| \rightarrow\left|\mathcal{K}_{n}^{\prime}\right| \text {. }
$$

Lemma 7.1. Each $f_{n}$ is $K$-quasiconformal, where $K$ is independent of $n$.

Proof. The lemma follows directly from the Ring Lemma and Lemma 6.2.

Of course, the most important feature we would expect from these discrete maps is some analog of the classical welding property $f\left(z^{-}\right)=f \varphi\left(z^{+}\right)$. By gluing vertices of $\gamma_{n}^{+}$to their images under the welding map $M_{n}^{-1} \varphi_{\tau_{\infty}, \tau^{\prime}}^{\epsilon} M_{n}$, the construction itself ensures the welding property holds at vertices. On the edges, however, the analogy breaks down somewhat. Our discrete conformal maps $f_{n}$ are affine on the edges, while the welding map clearly is not. Thus we need to form a discretized welding map which will respect the affine nature of $f_{n}$. To this end, we let

$$
\varphi_{n}(z)=M_{n}^{-1} \varphi_{\tau_{\infty}, \tau^{\prime}}^{\epsilon} M_{n}(z)
$$

if $z$ is a vertex and extend $\varphi_{n}$ affinely on the edges. That is, if $z$ lies on an edge $[v, w]$, we can write $z=(1-t) v+t w$ for some $t \in[0,1]$. Then we let

$$
\varphi_{n}(z)=(1-t) M_{n}^{-1} \varphi_{\tau_{\infty}, \tau^{\prime}}^{\epsilon} M_{n}(v)+t M_{n}^{-1} \varphi_{\tau_{\infty}, \tau^{\prime}}^{\epsilon} M_{n}(w)
$$

With this definition, the following lemma is immediate.

Lemma 7.2. For all $z \in \gamma_{n}, f_{n}\left(z^{-}\right)=f_{n} \varphi_{n}\left(z^{+}\right)$

Our notation is also obviously meant to suggest $\varphi_{n} \rightarrow \varphi_{\tau_{\infty}, \tau^{\prime}}^{\epsilon}$. We will see that this suggestion is indeed true, with one small caveat. Since the domain 
$\gamma_{n}$ of each $\varphi_{n}$ changes with $n$, we must understand convergence of $\varphi_{n}$ to mean convergence of the maps $\varphi_{n} M_{n}^{-1}: \gamma \rightarrow \gamma_{n}^{\prime}$.

Lemma 7.3. As $n \rightarrow \infty$, the meshes of both $P_{n}$ and $P_{n}^{\prime}$ decrease to 0 .

Proof. Recall that by Lemma 5.2, the points $\tau_{n}$ lie in a small ball about $\tau$. Thus each packing $P_{n}$ lifts to a packing in $\Omega_{\tau_{n}}$ lying near $\Omega_{\tau}$. In particular, all these fundamental parallelograms are contained inside a disc of some fixed radius. A standard application of Rodin and Sullivan's Length-Area Lemma [22] now shows that the mesh of $P_{n}$ must decrease to 0 .

Next observe that $f_{n}$ lifts to a quasiconformal map from $\Omega_{\tau_{n}}$ to $\Omega_{\tau_{n}^{\prime}}$. Since there is a uniform bound on the dilatation of the maps $f_{n}$, there is a uniform bound on the moduli of the fundamental parallelograms for $R_{\tau_{n}^{\prime}}$. This implies all these parallelograms lie in a disc of some fixed radius, and again we appeal to the Length-Area Lemma.

Lemma 7.4. As $n \rightarrow \infty, \varphi_{n} M_{n}^{-1} \rightarrow \varphi_{\tau_{\infty}, \tau^{\prime}}^{\epsilon}$ uniformly.

Proof. Since $M_{n}$ converges to the identity map, the lemma clearly holds on vertices of $\gamma_{n}$. If $M_{n}^{-1}(z)$ lies on an edge $[v, w]$ of $\gamma_{n}$, then

$\left|\varphi_{n} M_{n}^{-1}(z)-\varphi_{\tau_{\infty}, \tau^{\prime}}^{\epsilon}(z)\right| \leq\left|\varphi_{n} M_{n}^{-1}(z)-\varphi_{n} M_{n}^{-1}(v)\right|+$

$$
\left|\varphi_{n} M_{n}^{-1}(v)-\varphi_{\tau_{\infty}, \tau^{\prime}}^{\epsilon}(v)\right|+\left|\varphi_{\tau_{\infty}, \tau^{\prime}}^{\epsilon}(v)-\varphi_{\tau_{\infty}, \tau^{\prime}}^{\epsilon}(z)\right| .
$$

The second term can be made small since $v$ is a vertex; the first and third terms must be small by Lemma 7.3 and continuity.

7.5. Extending our maps. To show the convergence of our discrete conformal maps $f_{n}$, we will rely on a normal families result for quasiconformal maps of $\mathbb{C}$. However, to apply this result, we must lift each $f_{n}$ to a map $\widetilde{f}_{n}$ on the fundamental parallelogram $\Omega_{\tau_{n}} \subset \mathbb{C}$. More importantly, we must then extend $\widetilde{f}_{n}$ from $\Omega_{\tau_{n}}$ to all of $\mathbb{C}$.

First notice that $\gamma_{n}$ lifts to the line segment connecting 0 and $\tau$, but $\gamma_{n}$ also lifts to a line segment connecting 1 and $\tau+1$. We will consider this first lift to correspond to $\gamma_{n}^{+}$and label it $\widetilde{\gamma}_{n}^{+}$. Similarly, we view the second path $\widetilde{\gamma}_{n}^{-}$as a lift of $\gamma_{n}^{-}$. Now we view the lift $\widetilde{\varphi}_{n}$ of $\varphi_{n}$ as a map from $\widetilde{\gamma}_{n}^{+}$to $\widetilde{\gamma}_{n}^{-}$.

Notice that to extend $\tilde{f}$ across $\widetilde{\gamma}_{n}^{+}$, we cannot simply copy the action of $\tilde{f}$ to the adjacent fundamental parallelogram $\Omega_{\tau_{n}}-1$; that is, we cannot merely set $\widetilde{f}_{n}(z)=\widetilde{f}_{n}(z+1)-1$. Since the boundary values of $\widetilde{f}_{n}$ on $\widetilde{\gamma}_{n}^{+}$and $\widetilde{\gamma}_{n}^{-}$differ by $\widetilde{\varphi}_{n}$, such an extension could never be continuous on $\widetilde{\gamma}_{n}^{+}$. Thus before we attempt to repeat $\widetilde{f}_{n}$ on $\Omega_{\tau_{n}}-1$, we must first prepare the right side of $\Omega_{\tau_{n}}-1$ to mesh with the action of $\widetilde{f}_{n}$ on the left side of $\Omega_{\tau_{n}}$.

Our solution is to apply a self-map of $\Omega_{\tau_{n}}-1$ which agrees with $\widetilde{\varphi}_{n}^{-1}$ on the right side, but is the identity on the left side. If $\ell$ is a horizontal line segment through points $z$ on $\widetilde{\gamma}_{n}^{+}$(the right side of $\Omega_{\tau_{n}}-1$ ) and $z-1$ on the left side of $\Omega_{\tau_{n}}-1$, then we define $\widetilde{\Phi}_{n} \mid \ell$ to be the affine map sending $\ell$ to 
the line segment joining $\widetilde{\varphi}_{n}(z)$ and $z-1$. See Figure 13. It is not difficult to see that $\widetilde{\Phi}_{n}$ is quasiconformal with dilatation depending only on $\widetilde{\varphi}_{n}$. But Lemma 7.4 then implies the entire family $\left\{\widetilde{\Phi}_{n}\right\}$ is uniformly quasiconformal with dilatation depending only on $\epsilon$ and $\tau_{\infty}$.

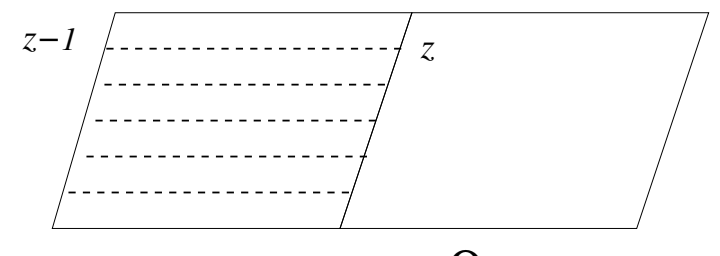
$\Omega_{n}-1$
$\Omega_{n}$

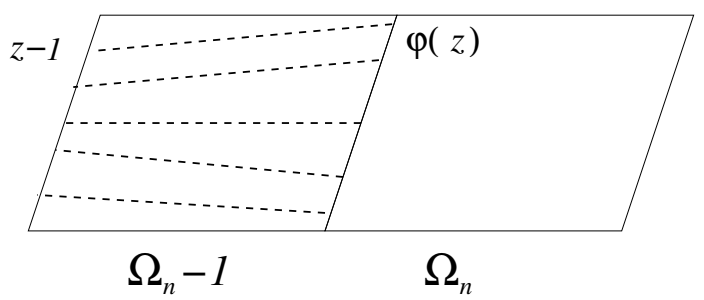

Figure 13. We extend $\varphi_{n}$ to all of $\Omega_{\tau_{n}}-1$ by acting on horizontal geodesics with endpoints on $\gamma_{n}^{+}$.

Notice that since $0,1, \tau$, and $\tau+1$ all correspond to the fixed point $v$ of $\varphi_{n}, \widetilde{\Phi}_{n}$ is the identity map on the top and bottom of the parallelogram $\Omega_{\tau_{n}}-1$.

If $z \in \widetilde{\gamma}_{n}^{+}$, then $\widetilde{\Phi}_{n}^{-1}(z)+1 \in \widetilde{\gamma}_{n}^{-}$. Thus by Lemma 7.2 (assuming here and throughout a compatible choice of liftings $\widetilde{f}_{n}$ and $\left.\widetilde{\varphi}_{n}\right)$,

$$
\begin{aligned}
\tilde{f}_{n}\left(\widetilde{\Phi}_{n}^{-1}(z)+1\right)-1 & =\widetilde{f}_{n}\left(\widetilde{\varphi}_{n}^{-1}(z)+1\right)-1 \\
& =\widetilde{f}_{n} \widetilde{\varphi}_{n}\left(\widetilde{\varphi}_{n}^{-1}(z)\right) \\
& =\widetilde{f}_{n}(z) .
\end{aligned}
$$

As a result, we can continuously extend $\widetilde{f}_{n}$ across $\widetilde{\gamma}_{n}^{+}$. On the fundamental parallelogram $\Omega_{\tau_{n}}-1$ lying to the left of $\widetilde{\gamma}_{n}^{+}$, we define

$$
\tilde{f}_{n}(z)=\tilde{f}_{n}\left(\widetilde{\Phi}_{n}^{-1}(z)+1\right)-1 .
$$

Since $\widetilde{\Phi}_{n}$ was the identity on the left side of $\Omega_{\tau_{n}}-1$, our extension of $\widetilde{f}_{n}$ reduces to $\widetilde{f}_{n}(z+1)-1$ there. That is, both $\widetilde{f}_{n}$ restricted to the left side of $\Omega_{\tau_{n}}-1$ and $\widetilde{f}_{n} \mid \widetilde{\gamma}_{n}^{+}$are lifts of $f_{n} \mid \gamma_{n}^{+}$. Consequently, we may propagate our extensions periodically on $\Omega_{\tau_{n}}-2, \Omega_{\tau_{n}}-3$, and so forth. 
Similarly, on the fundamental parallelogram $\Omega_{\tau_{n}}+1$ lying to the right of $\widetilde{\gamma}_{n}^{-}$, we define

$$
\widetilde{f}_{n}(z)=\widetilde{f}_{n} \widetilde{\Psi}_{n}(z-1)+1
$$

where $\widetilde{\Psi}_{n}$ is the extension of $\widetilde{\varphi}_{n}$ to $\Omega_{\tau_{n}}$ which sends line segments joining $z \in \widetilde{\gamma}_{n}^{+}$and $z+1$ to line segments joining $\widetilde{\varphi}_{n}(z)$ and $z+1$. The family $\left\{\widetilde{\Psi}_{n}\right\}$ will be uniformly quasiconformal with dilation depending only on $\epsilon$ and $\tau_{\infty}$.

Again observe that if $z \in \widetilde{\gamma}_{n}^{-}$, then $\widetilde{\Psi}_{n}(z-1) \in \widetilde{\gamma}_{n}^{+}$. Hence

$$
\begin{aligned}
\widetilde{f}_{n}\left(\widetilde{\Psi}_{n}(z-1)\right)+1 & =\widetilde{f}_{n}\left(\widetilde{\varphi}_{n}(z-1)\right)+1 \\
& =\widetilde{f}_{n}(z),
\end{aligned}
$$

and $\widetilde{f}_{n}$ is continuous across $\widetilde{\gamma}_{n}^{-}$. As before, we may extend $\widetilde{f}_{n}$ periodically to the right.

Finally we extend $\widetilde{f}_{n}$ vertically by conjugation with the covering transformations $z \mapsto z \pm m \tau_{n}, m=1,2, \ldots, \infty$. Since the covering transformations, $\widetilde{\Phi}_{n}, \widetilde{\Psi}_{n}$, and $f_{n}$ are all uniformly quaisiconformal, the maps $\widetilde{f}_{n}$ will be uniformly quasiconformal on all of $\mathbb{C}$.

7.6. Arriving at a proof. Our newly extended maps now lead us to a proof of Theorem 1.2.

Theorem 1.2. Fix any abstract triangulation $\mathcal{K}$ of a torus and any point $\tau^{\prime} \in \mathbb{H}$. Then combinatorially deforming $\mathcal{K}$ by hex refinement and combinatorial welding along an appropriate geodesic produces packable surfaces which converge in the Teichmüller metric to $\tau^{\prime}$.

Proof. Each $\widetilde{f}_{n}$ is a quasiconformal map of $\mathbb{C}$ onto itself which fixes $\mathbb{Z}$. The dilatations of these maps are uniformly bounded. Thus by a standard normal families argument [16], a subsequence converges uniformly on compact subsets of $\mathbb{C}$ to a quasiconformal homeomorphism $\tilde{f}$. In particular, since $\tau_{n} \rightarrow \tau_{\infty}$, a subsequence converges uniformly on a compact set which contains each parallelogram $\Omega_{\tau_{n}}$. We will temporarily re-number and assume the entire sequence $\left\{\widetilde{f}_{n}\right\}$ converges uniformly there.

It follows from the Packing Lemma that $\widetilde{f}$ restricted to $\Omega_{\tau_{\infty}}$ is conformal. Moreover, since each $\widetilde{f}_{n}$ was extended by conjugation by the covering transformations $z \mapsto z \pm m \tau_{n}$, each $\widetilde{f}_{n}$ projects to $R_{\tau_{n}}$, and $\widetilde{f}$ projects to a map $f$ on $R_{\tau_{\infty}}$.

Since $M_{n}$ converges uniformly to the identity, Lemma 7.4 implies

$$
f_{n} \varphi_{n} M_{n}^{-1}\left(z^{+}\right) \rightarrow f \varphi_{\tau_{\infty}, \tau^{\prime}}^{\epsilon}\left(z^{+}\right)
$$

uniformly on $\gamma_{n}^{+}$.

But by Lemma 7.2,

$$
f_{n} \varphi_{n} M_{n}^{-1}\left(z^{+}\right) \rightarrow f\left(z^{-}\right) .
$$


Combining (7.3) and (7.4), we see $f$ satisfies the welding condition

$$
f \varphi_{\tau_{\infty}, \tau^{\prime}}^{\epsilon}\left(z^{+}\right)=f\left(z^{-}\right)
$$

on $\gamma$. Thus $f$ is a conformal welding for $\varphi_{\tau_{\infty}, \tau^{\prime}}^{\epsilon}$. By the Theorem 3.2 [20], $R_{\tau_{\infty}}$ must then be the unique surface determined by $\varphi_{\tau_{\infty}, \tau^{\prime}}^{\epsilon}$

Notice that any subsequence of $\left\{f_{n}\right\}$ would satisfy the normality conditions and the welding conditions in the limit. Thus every subsequence of the original $\left\{f_{n}\right\}$ converges to the unique welding $f$. Consequently, the entire original sequence must converge.

Finally, we let $\epsilon \rightarrow 0$. The theorem follows by a standard diagonalization argument.

7.7. Density in Teichmüller space. Not only are the packable tori dense [8], but any one complex $K$ generates a dense packable subset. This is even more surprising in light of the fact that aside from the hex refinements which occur evenly over the complex, welding essentially changes the combinatorics of $\mathcal{K}$ only near a fixed geodesic.

Corollary 7.5. Let $K$ be any abstract triangulation of a torus. The set of all points in moduli space which support a packing for a welding deformation of $\mathcal{K}$ is dense.

7.8. Approximating any bilipschitz welding. Finally notice that the proof of Theorem 1.2 can be applied to any bilipschitz welding map, not just the special ones defined in Section 3.2. Thus our discrete welding provides a means to compute the heretofore unknown target surface for a given bilipschitz welding map.

Corollary 7.6. Let $R_{\tau}$ be any torus, $\gamma \subset R_{\tau}$ a simple closed geodesic, and $\varphi: \gamma \rightarrow \gamma$ a bilipschitz homeomorphism with a fixed point $v$. Then there is a sequence of packable welded tori converging in the Teichmüller metric to a surface $R_{\tau^{\prime}}$ obtainable from $R_{\tau}$ by welding by $\varphi$.

\section{References}

[1] D. Aharonov, The sharp constant in the ring lemma, Complex Variables Theory Appl., 33 (1997), 27-31, MR 99c:30008, Zbl 0903.30006.

[2] D. Aharonov and K. Stephenson, Geometric sequences of discs in the Apollonian packing, Algebra i Analiz, 9 (1997), 104-140, MR 98f:52019, Zbl 0908.52006.

[3] R.W. Barnard and K. Pearce, Rounding corners of gearlike domains and the omitted area problem, J. Comput. Appl. Math., 14 (1986), 217-226, MR 87f:30014, Zbl 0593.30012.

[4] A.F. Beardon and K. Stephenson, The uniformization theorem for circle packings, Indiana Univ. Math. J., 39 (1990), 1383-1425, MR 92b:52038, Zbl 0797.30008. 
[5] P.L. Bowers and K. Stephenson, Uniformizing dessins and Belyı maps via circle packing, submitted.

[6] - The set of circle packing points in the Teichmüller space of a surface of finite conformal type is dense, Math. Proc. Camb. Phil. Soc., 111 (1992), 487-513, MR 93a:30050, Zbl 0756.30036.

[7] _ Circle packings in surfaces of finite type: An in situ approach with application to moduli, Topology, 32 (1993), 157-183, MR 94d:30083, Zbl 0783.30030.

[8] R. Brooks, Circle packings and co-compact extensions of Kleinian groups, Inventiones Mathematicae, 86 (1986), 461-469, MR 88b:32050, Zbl 0593.30048.

[9] T.A. Driscoll, Algorithm 756: A MATLAB Toolbox for Schwarz-Christoffel mapping, ACM Trans. Math. Softw., 22(2) (1996), 168-186, Zbl 0884.30005; software available at http://amath.colorado.edu/appm/faculty/tad/research/sc.html.

[10] F.P. Gardiner, Teichmüller Theory and Quadratic Differentials, Wiley, 1987, MR 88m:32044, Zbl 0629.30002.

[11] F.P. Gardiner and N. Lakic, Quasiconformal Teichmüller Theory, Mathematical Surveys and Monographs, 76, American Mathematical Society, 2000, MR 2001d:32016, Zbl 0949.30002.

[12] Z.-X. He and B. Rodin, Convergence of circle packings of finite valence to Riemann mappings, Comm. in Analysis and Geometry, 1 (1993), 31-41, MR 94m:30019, Zbl 0777.30003.

[13] Y. Imayoshi and M. Taniguchi, An Introduction to Teichmüller Spaces, SpringerVerlag, 1992, MR 94b:32031, Zbl 0754.30001.

[14] Y. Katznelson, S. Nag and D.P. Sullivan, On conformal welding homeomorphisms associated to Jordan curves, Ann. Acad. Sci. Fenn. Ser. A I Math., 15 (1990), 293306, MR 92e:30009, Zbl 0688.30003.

[15] O. Lehto, Univalent Functions and Teichmüller Spaces, Springer-Verlag, 1987, MR 88f:30073, Zbl 0606.30001.

[16] O. Lehto and K.I. Virtanen, Quasiconformal Mappings in the Plane, Springer-Verlag, Berlin-Heidelberg-New York, 1973, MR 49 \#9202, Zbl 0267.30016.

[17] D. Minda and B. Rodin, Circle packing and Riemann surfaces, J. D'Analyse Math., 57 (1991), 221-249, MR 94f:30056, Zbl 0770.52008.

[18] S. Nag, The Complex Analytic Theory of Teichmüller Spaces, Wiley, 1988, MR 89f:32040, Zbl 0667.30040.

[19] _ Singular Cauchy integrals and conformal welding on Jordan curves, Ann. Acad. Sci. Fenn. Ser. A I Math., 21 (1996), 81-88, MR 97c:30010, Zbl 0846.30006.

[20] K. Oikawa, Welding of polygons and the type of Riemann surfaces, Kodai Math. Sem. Rep., 13 (1961), 37-52, MR 23 \#A3253, Zbl 0129.05702.

[21] D. Partyka, A Sewing Theorem for complementary Jordan domains, Annales Universitatis Mariae Curie-Sklodowska-Lublin-Polonia, 16 (1987), 99-103, MR 91e:30043, Zbl 0698.30016.

[22] B. Rodin and D. Sullivan, The convergence of circle packings to the Riemann mapping, J. Differential Geometry, 26 (1987), 349-360, MR 90c:30007, Zbl 0694.30006.

[23] K. Stephenson, A Probabilistic proof of Thurston's conjecture on circle packings, Rend. Sem. Mat. Fis. Milano, 66 (1996), 201-291, MR 99m:52024. 
[24] W. Thurston, The Geometry and Topology of 3-Manifolds, Princeton University Notes, preprint.

[25] _ The finite Riemann mapping theorem, Invited talk, An International Symposium at Purdue University on the occasion of the proof of the Bieberbach conjecture, March 1985.

[26] M. Toki, Moduli of tori obtained by conformal sewing, preprint.

[27] G.B. Williams, Discrete Conformal Welding, preprint.

[28] _ Earthquakes and Circle Packings, J. Anal. Math., 85 (2001), 371-396, CMP 1869616.

[29] _ Approximating of quasisymmetries using circle packings, Discrete Comput. Geom., 25(1) (2001), 103-124, CMP 1797299.

Received October 31, 2000 and revised May 31, 2001.

Department of Mathematics

TeXas TeCh University

LUBBOCK, TX 79409

E-mail address: barnard@math.ttu.edu

Department of Mathematics

Texas Tech University

LUBBOCK, TX 79409

E-mail address: williams@math.ttu.edu 